\title{
Consumption of fruits and vegetables in relation to the risk of developing acute coronary syndromes; the CARDIO2000
} case-control study

\author{
Demosthenes B Panagiotakos*1,2, Christos Pitsavos¹, Peter Kokkinos², \\ Christina Chrysohoou ${ }^{1}$, Manolis Vavuranakis ${ }^{1}$, Christodoulos Stefanadis ${ }^{1}$ \\ and Pavlos Toutouzas ${ }^{1}$
}

Address: ${ }^{1}$ Department of Cardiology, School of Medicine, University of Athens, Greece and ${ }^{2}$ Veterans Affairs Medical Center and Cardiology Division, Georgetown University, Washington, DC, USA

Email: Demosthenes B Panagiotakos* - d.b.panagiotakos@usa.net; Christos Pitsavos - dbpanag@math.uoa.gr;

Peter Kokkinos - pfk3000@aol.com; Christina Chrysohoou - chrysohoou@usa.net; Manolis Vavuranakis - dbpanag@math.uoa.gr; Christodoulos Stefanadis - dbpanag@math.uoa.gr; Pavlos Toutouzas - dbpanag@math.uoa.gr

* Corresponding author

Published: 8 May 2003

Nutrition Journal 2003, 2:2

This article is available from: http://www.nutritionj.com/content/2/l/2

(C) 2003 Panagiotakos et al; licensee BioMed Central Ltd. This is an Open Access article: verbatim copying and redistribution of this article are permitted in all media for any purpose, provided this notice is preserved along with the article's original URL.

\begin{abstract}
Background: The relation between diet and human health has long been investigated. The aim of this work is to evaluate the association between CHD risk and the consumption of fruit and vegetable, in a large sample of cardiac patients and controls.

Methods: Stratified sampling from all Greek regions, consisted of 848 (700 males, $58 \pm 10$ years old and I 48 females, $65 \pm 9$ years old) randomly selected patients, admitted to the cardiology clinic for a first event of an acute coronary syndrome (ACS). In addition we selected 1078 frequency paired, by sex-age-region, controls in the same hospitals but without any clinical suspicion of CHD. Using validated food-frequency questionnaires we assessed total diet, including fruit and vegetable intake, on a weekly basis. Multiple logistic regression analysis estimated the relative risk of developing ACS by level of fruits and vegetables intake after taking into account the effect of several potential confounders.
\end{abstract}

Results: Data analysis revealed that the benefit of fruit or vegetable consumption increases proportionally by the number of servings consumed ( $P$ for trend $<0.00 \mathrm{I})$. After adjusting for the conventional cardiovascular risk factors, those in the upper quintile of fruit consumption $(5$ or more items/day) had $72 \%$ lower risk for $\mathrm{CHD}$ (odds ratio $=0.28,95 \% \mathrm{Cl} 0.1 \mathrm{I}-0.54, \mathrm{P}<0.00 \mathrm{I}$ ), compared with those in the lowest quintile of intake $(<1$ items/day). Similarly, consumption of vegetable more than 3 days / week was associated with $70 \%$ lower risk for $\mathrm{CHD}$ (odds ratio $=0.30$, $95 \% \mathrm{Cl} 0.22-0.40, \mathrm{P}<0.00 \mathrm{I}$ ), compared with those that they did not consume vegetables. Of particular interest, a $10 \%$ reduction in coronary risk was observed for every one piece of fruit consumed per day (odds ratio $=0.90,95 \% \mathrm{Cl} 0.85-0.97, \mathrm{P}=0.004$ ).

Conclusions: Consumption of fruits and vegetables seems to offer significant protection against CHD. 


\section{Background}

Coronary heart disease is one of the leading causes of death, universally and one of the most common chronic illnesses in many countries of the developing world $[1,2]$. Among the conventional cardiovascular risk factors, diet poses a particular challenge for research. Cross-cultural comparisons, case-control and prospective observational studies identified a relationship between diet, blood pressure and lipids levels [3-6], but there is still considerable scientific uncertainty about the relationship between specific dietary components and cardiovascular risk [7], especially in Mediterranean populations.

In this work we quantified the public health benefit of fruit and vegetable consumption on the primary prevention of acute coronary syndromes (acute myocardial infarction or unstable angina) using a large sample of cardiac patients and frequency-matched controls from all Greek regions.

\section{Methods}

The CARDIO2000 is a multicentre case-control study that explores the association between several demographic, nutritional, lifestyle, clinical and biochemical risk factors with the risk of developing non-fatal acute coronary syndromes. From January 2000 to March 2002, we randomly selected 848 coronary patients and 1078 cardiovascular symptom free subjects (controls). The number of the enrolled subjects was decided through power analysis, in order to evaluate (two sided) differences in the coronary relative risk greater than $7 \%$ with statistical power greater than $80 \%$ and significant level less than 5\%. According to the population distribution provided by the National Statistical Services (Ministry of Economics, census 1991), we stratified our sampling into all the Greek regions. Controls were mainly individuals who visited the outpatient departments of the same hospital at the same period with the coronary patients, for routine examinations or minor surgical operations. In a few cases (in country hospitals) where the available number of hospitalised controls was not sufficient for the matching procedure, we enrolled into the study friends or colleagues of the coronary patients. All participants were informed about the aims of the study and agreed to give the requested information. In order to eliminate recall bias we tried to retrieve precise information from cases and controls and medical history through hospital or the insurance records.

The coronary patients were selected from the admission listing of the cardiological clinics. According to the study's protocol the patients were hospitalised for an acute first event of coronary heart disease (acute myocardial infarction or unstable angina; stable angina was excluded from the analysis). The selection criteria for cardiac patients are:
- diagnosis of first acute myocardial infarction (MI). MI was defined by any two features: electrocardiographic changes, compatible clinical symptoms, and specific diagnostic enzyme elevations of SGOT, CPK, LDH and troponin T or I ( $49 \%$ of the patients had MI); or

- diagnosis of unstable angina (i.e. one or more angina episodes at rest within the preceding 48 hours) corresponding to class III of the Braunwald classification (51\% of the patients had unstable angina).

Afterwards, we randomly selected subjects without any clinical symptoms, signs or suspicions of cardiovascular disease in their medical history (controls), frequently matched to the patients by age ( \pm 3 years), sex, and region. The CARDIO2000 investigators performed the evaluation of clinical symptoms and signs in the series of cases and controls.

The information regarding the investigated medical factors was retrieved from the subjects' medical records, along with lifestyle characteristics, through a confidential, detailed questionnaire administered during Physician interview after the second day of hospitalisation, for cases and at entry for controls. The subjects social status was investigated through educational and financial stratification. In particular, the educational level was classified into three groups: Group I: education through high school $(0$ - 9 years of schooling); Group II: high school, technical education up to 14 years of schooling (but not academic) and Group III: (university). Current smokers were defined as those who smoked at least one cigarette per day. Former smokers were those who had stopped smoking for at least one year, and never smokers those who never smoked a cigarette in their lives. Those who stopped smoking for less than a year were classified as smokers. Quantification of smoking status was based on the calculation of packyears adjusted for nicotine content equal to $0.8 \mathrm{mg}$ per cigarette. Physical activity was defined as any type of nonoccupational physical exercise, at least once/week during the past year, and was graded in qualitative terms such as light (expended calories $<4 \mathrm{Kcal} / \mathrm{min}$, i.e. walking slowly, stationary cycling, light stretching etc.), moderate (expended calories 4-7 Kcal/min, i.e. walking briskly, outdoor cycling, swimming moderate effort etc.) and vigorous (expended calories $>7 \mathrm{Kcal} / \mathrm{min}$, i.e. walking briskly uphill, long distance running, cycling fast or racing, swimming fast crawl etc.). The rest of the subjects were defined as physically inactive. Moreover, since physical activity is an important factor for cardiovascular disease, we took into account the occupational activity status of all participants.

The evaluation of the nutritional habits was accomplished with a validated questionnaire based on the guidelines 
from the Department of Nutrition of the National School of Public Health [8]. We measured the consumption of several food items as an average per week during the past year. The frequency of consumption was quantified approximately in terms of the number of times per month this food was consumed. Thus, daily consumption multiplied by 30 and weekly consumption multiplied by 4 and a value of 0 was assigned to food items rarely or never consumed. In order to describe overall diet we used composite scores, which are necessary for the evaluation of epidemiological associations. Specifically for fruit and vegetable intake the participants reported their average consumption of a specific portion size for these foods over the past year, including apples, grapes, pears, bananas, oranges, etc, as well as broccoli, carrots, spinach, lettuce salad, yellow squash, and tomatoes. Vegetable intake was categorized into $<1$ serving/day, $1-1.49$ servings/day, 1.5 - 1.99 servings/day, 2 - 2.49 servings/day, and 2.5+ servings/day, based both on maintaining a natural gradient of exposure and including adequate participants in each category. The provided list of fruits and vegetables was comprehensive enough to capture most types of fruits and vegetables that our subjects consumed. Although fruit juice was recorded it was not evaluated due to low consumption $(<5 \%)$. Alcohol, a potential confounder for total energy intake, was measured by daily ethanol consumption (red or white wine, beer, and other beverages), in wineglasses of $100 \mathrm{ml}$ and $12 \%$ ethanol concentration) as done by other investigators [9].

According to the collected medical information, the majority of the controls $(86 \%)$ and the patients $(83 \%)$ had at least one laboratory measurement during the past 12 months. In addition, we measured, both in patients and controls, arterial blood pressure levels in the right arm (average of 3 measurements having the patient seated and rested), total cholesterol and fasting glucose levels. For the cardiac patients the measurements were collected during the first $12 \mathrm{~h}$ of hospitalization and at the end of the interview for the controls. The previous information as well as the patients' and controls' reports assisted us in characterizing the subjects as having hypertension, hypercholesterolemia, or diabetes. In keeping with the long-standing classification criteria used in several population-based studies, patients whose blood pressure, according to their medical records, were greater or equal to $140 / 90 \mathrm{mmHg}$ or were taking antihypertensive medication were classified as hypertensives. Hypercholesterolemia was defined as cholesterol levels greater than $220 \mathrm{mg} / \mathrm{dl}$ or greater than $200 \mathrm{mg} / \mathrm{dl}$ when two other risk factors for coronary heart disease were present or if hypo-lipidemic treatment was administered. Diabetics were those with fasting blood glucose greater than $125 \mathrm{mg} / \mathrm{dl}$ or those who were under special diet or treatment. Finally, we measured the height and the weight both in patients and controls and we calculated the body mass index $\left(\mathrm{BMI}=\right.$ weight $\left./\{\text { height }\}^{2}\right)$. In addition, we asked for any significant changes in their body mass during the past years. Obesity was defined as BMI > $29.9 \mathrm{Kg} / \mathrm{m}^{2}$. Presence of premature coronary heart disease among first-degree relatives $(<55$ years for male relatives and $<65$ for female relatives) was also recorded. Further details regarding the aims, design and methodology of the CARDIO2000 study have been previously presented [10-13].

\section{Statistical analysis}

Continuous variables are presented as mean \pm one standard deviation, while qualitative variables are presented as absolute and relative frequencies. In order to fit a multivariate risk model that evaluates the investigated parameters on the risk of developing acute coronary syndromes, an exploratory analysis was initially applied. In particular, stratified by sex contingency tables were conducted in order to investigate associations between categorical variables and groups of study, by the calculation of the overall chi-squared criterion. Also, Wilcoxon non-parametric criterion was applied in order to evaluate differences in continuous measurements (i.e. body mass index) between groups. Estimations of the relative risks of developing acute coronary syndromes were performed by the calculation of the odds ratio (OR) and the corresponding confidence intervals through multiple conditional logistic regression analysis, adjusting for age (in years), body mass index (BMI) $\left(\mathrm{kg} / \mathrm{m}^{2}\right)$, smoking, alcohol intake, physical activity, history of high cholesterol or hypertension or diabetes or premature CHD, and use of multivitamins. We then conducted a stratified analysis according to smoking status (past, current, never) to evaluate whether the relation between fruits and vegetable intakes and CHD risk differed by these variables. Tests of linear trend across increasing categories of vegetable consumption were conducted by assigning the medians of intakes in categories (servings / day) treated as a continuous variable.

All reported P-values are from two-sided tests and compared to a significant level of 5\%. STATA 6 software was used for the calculations (STATA Corp. College Station, Texas, USA).

\section{Results}

Seven hundred $(83 \%)$ of the coronary patients were males and $148(17 \%)$ were females, while $862(80 \%)$ of the controls were males and $216(20 \%)$ were females. Table 1 describes the characteristics of the patients and the controls. The data analysis revealed that $365(43 \%)$ of the cardiac patients and $722(67 \%)$ of the controls consumed some quantity of fruit and vegetable daily. Of these, $28(3 \%)$ patients and $15(1 \%)$ controls reported consuming < 1 serving/day; $351(41 \%)$ patients and $377(35 \%)$ controls reported consuming $1-1.49$ servings/day; 344 (40\%) 
Table I: Characteristics of the study's population

\begin{tabular}{|c|c|c|c|c|c|c|c|c|c|}
\hline & \multicolumn{4}{|c|}{ ACS Patients } & \multicolumn{5}{|l|}{ Controls } \\
\hline & Males & & Females & & Males & & Females & & P-value \\
\hline Number & $700(82 \%)$ & & 148 (I8\%) & & $862(80 \%)$ & & $216(20 \%)$ & & \\
\hline Age (years) & $59.1 \pm 10$ & & $65.3 \pm 9$ & & $58.8 \pm 10$ & & $64.8 \pm 10$ & & \\
\hline Education & & & & & & & & & 0.012 \\
\hline Group I (0 - 9 yrs) & 406 & $58 \%$ & 114 & $77 \%$ & 474 & $55 \%$ & 147 & $68 \%$ & \\
\hline Group II (10-14 yrs) & 182 & $26 \%$ & 31 & $21 \%$ & 198 & $23 \%$ & 41 & $19 \%$ & \\
\hline Group III (> I 4 yrs) & 112 & $16 \%$ & 3 & $2 \%$ & 190 & $22 \%$ & 28 & $13 \%$ & \\
\hline Current smoking & 525 & $75 \%$ & 44 & $30 \%$ & 500 & $58 \%$ & 54 & $25 \%$ & $<0.001$ \\
\hline Pack years & $39.9 \pm 14$ & & $13.1 \pm 9$ & & $21.5 \pm 13$ & & $5.2 \pm 6$ & & $<0.001$ \\
\hline Hypertension & 308 & $44 \%$ & 102 & $69 \%$ & 216 & $25 \%$ & 69 & $32 \%$ & $<0.001$ \\
\hline Hypercholesterolemia & 413 & $59 \%$ & 40 & $27 \%$ & 586 & $68 \%$ & 67 & $31 \%$ & $<0.001$ \\
\hline Diabetes mellitus & 168 & $24 \%$ & 15 & $10 \%$ & 267 & $31 \%$ & 17 & $8 \%$ & $<0.001$ \\
\hline Family history of CHD & 308 & $44 \%$ & 77 & $52 \%$ & 129 & $15 \%$ & 39 & $18 \%$ & $<0.001$ \\
\hline Body mass index $\left(\mathrm{Kg} / \mathrm{m}^{2}\right)$ & $27.4 \pm 4$ & & $27.1 \pm 4$ & & $27.1 \pm 3$ & & $26.7 \pm 2$ & & 0.120 \\
\hline Physical activity & 252 & $36 \%$ & 37 & $25 \%$ & 371 & $43 \%$ & 84 & $39 \%$ & 0.003 \\
\hline Alcohol consumption & 336 & $48 \%$ & 25 & $17 \%$ & 379 & $44 \%$ & 30 & $14 \%$ & 0.024 \\
\hline
\end{tabular}

Comparisons were based on the chi-squared test and the Wilcoxon criterion. P-value is for group differences after controlling for gender.

patients and $428(40 \%)$ controls reported 1.5 - 1.99 servings/day; 100 (9\%) patients and 150 (14\%) controls reported $2-2.49$ servings/day, and $33(7 \%)$ patients and $100(10 \%)$ controls consumed $2.5+$ servings/day ( $P$ for trend $<0.001)$. We then examined the association between the consumption of fruit, or vegetable and the prevalence of hypertension, diabetes mellitus, hypercholesterolemia, as well as cigarette smoking, body mass index, and physical inactivity, since these factors are considered modifiers of the effect of fruits and vegetables on coronary risk [9]. After stratifying our analysis by group of study (patients vs. controls), an inverse association revealed between fruit and vegetable consumption and cigarette smoking (P-value $=0.008, \mathrm{P}$-value $=0.04$, respectively), prevalence of hypertension (P-value = 0.038 , and $P=0.067$, respectively), and diabetes mellitus $(\mathrm{P}=0.044$, and $\mathrm{P}$-value $=0.04$, respectively $)$. Furthermore, a positive association was found between fruit and vegetable consumption and education level both in patients and controls (P-value $=0.042, \mathrm{P}$-value $=0.001$, respectively). No significant associations were observed between fruit and vegetables intake and the prevalence of hypercholesterolemia $(\mathrm{P}=0.574, \mathrm{P}$-value $=0.804$, respectively), obesity $(\mathrm{P}$-value $=0.579, \mathrm{P}$-value $=0.565$, respectively), alcohol consumption $(\mathrm{P}$-value $=0.939$, $\mathrm{P}$-value $=$ 0.919 , respectively) and physical inactivity status (P-value $=0.384, \mathrm{P}$-value $=0.067$, respectively) . After adjusting for the aforementioned risk factors, those in the upper quintile of fruit consumption (5 or more items /day) had $72 \%$ lower relative risk for $\mathrm{CHD}(\mathrm{OR}=0.28,95 \%$ CI 0.11 $0.54)$, compared with those in the lowest quintile of intake ( $<1$ item/day). Similarly, consumption of vegetable more than 3 days / week was associated with 70\% lower relative risk for CHD (odds ratio $=0.30,95 \%$ CI 0.22 0.40 ), compared with those that they did not consume vegetables. Of particular interest, a $10 \%$ reduction in coronary risk was observed for every additional piece of fruit consumed per day (OR $=0.90,95 \%$ CI $0.85-0.97)$, after controlling for the conventional cardiovascular risk factors. Adjusting for the same covariates in an analysis of the overall trend, considering vegetable intake as a continuous variable, we found an odds ratio of 0.81 (95\% CI 0.67 - 0.93) for developing CHD per each additional serving / day of vegetable consumed. The individual effect of fruit and vegetable consumption on coronary risk is presented in Table 2. Moreover, green vegetable intake showed a $12 \%$ reduction on coronary risk per item/day (odds ratio $=0.88$, 95\% CI 0.77 - 0.97), and vitamin C-rich fruits showed an $11 \%$ reduction per item/day (odds ratio = $0.89,95 \%$ CI $0.84-0.95)$.

We then stratified the previous analysis by smoking status (current vs. never / former smokers) and found that the protective effect of fruit and vegetable consumption on coronary risk is diminished in the subgroup of heavy $(+31$ cigarettes per day) current smokers. However, some protection appears to be provided. More specifically, consumption of $2.5+$ servings of vegetable per day is associated with a $27 \%$ lower risk of developing acute coronary syndromes compared to those who consumed $<1$ serving per day (odds ratio $=0.73,95 \%$ CI $0.27-1.96$ ). Moreover, each 1-piece/day increase of fruit consumption is associated with 5\% lower coronary risk in current smokers (odds ratio $=0.95,95 \%$ CI $0.86-1.04$ ).

\section{Discussion}

The present study revealed that even a moderate consumption of fruit and vegetable $1-2$ servings per day) is associated with significantly lower risk of coronary events, even after controlling for several potential confounding 
Table 2: Results from the multivariate logistic model that was developed for the evaluation of the effect of fruits and vegetables consumption on coronary risk

\begin{tabular}{|c|c|c|c|c|}
\hline \multirow[b]{2}{*}{ Variable } & \multirow[t]{2}{*}{ OR } & \multicolumn{2}{|c|}{$95 \%$ C.I. for OR } & \multirow[t]{2}{*}{ P-Value } \\
\hline & & Lower & Upper & \\
\hline Age (years) & 1.015 & 1.006 & 1.025 & 0.001 \\
\hline Female gender & 0.402 & 0.320 & 0.505 & $<0.001$ \\
\hline \multicolumn{5}{|l|}{ Vegetable consumption } \\
\hline$<<$ serving / day & 1.00 & - & - & - \\
\hline I - I.49 servings / day & 0.562 & 0.387 & 0.901 & 0.012 \\
\hline $1.5-1.99$ servings / day & 0.367 & 0.189 & 0.712 & 0.003 \\
\hline $2-2.49$ servings / day & 0.297 & 0.150 & 0.588 & $<0.001$ \\
\hline $2.5+$ servings / day & 0.281 & 0.110 & 0.541 & $<0.001$ \\
\hline Fruit consumption (per item) & 0.904 & 0.845 & 0.967 & 0.004 \\
\hline
\end{tabular}

The odds ratios were estimated after taking into account the effect of hypertension, hypercholesterolemia, diabetes mellitus, family history of premature coronary heart disease, physical activity status, smoking habits, body mass index, alcohol consumption, and education status of the participants.

risk factors (Table 2). Furthermore, the risk was progressively lower as the consumption of fruits or vegetables increased. Those in the highest quintile of consumption ( 2.5 or more serving per day) had about $70 \%$ lower relative risk when compared to those in the lowest quintile. Moreover, some protection appears to be provided even in the group of current smokers.

Our findings are in accordance with those of previous epidemiological studies, which concluded that the consumption of fruits and vegetables might reduce disease risk, particularly for cancer and cardiovascular disease $[9,14,15]$. However, less is known about the direct association between fruits and vegetable intake and risk of acute coronary syndromes $[9,15]$. Nine of ten ecological studies two out of three case-control studies and six of sixteen cohort studies reported a significant protective association of CHD with consumption of fruit and vegetables [5]. Moreover, a review of 250 observational studies reported that increased consumption of fruits and vegetables could reduce cardiovascular deaths from $6 \%$ to $22 \%(8,000$ deaths annually) [16]. Our findings support that even low consumption of fruits and vegetables (1-2 servings per week) is associated with about $45 \%$ lower coronary risk (Table 2). Consumption of 2 or more servings per week is associated with about $70 \%$ reduction in relative risk. This translates to a considerable prevention of acute coronary events, carries a large public health potential, and emphasises the importance of following dietary recommendations as a whole. Moreover, our findings on smokers and the consumption of fruits and vegetables are worth mentioning. Although statistical significance was not achieved, a trend was evident, suggesting that the consumption of fruits and vegetables may offer some protection for current smokers. Since smokers consume substantially less fruit and fruit juice then non-smokers with heavy smokers eating the least [17], special attention should be given to dietary interventions for smokers in order to improve their nutrition. However, smoking cessation must remain the primary target for CHD prevention. A discussion of the mechanisms responsible for the protection fruit and vegetable consumption offers is beyond the scope of this study. However, several investigators reported that the antioxidant, potassium, fiber, and folate content of fruits and vegetables could at least partially explain their protective effect of CHD [18-20]. Other compounds such as flavonoids, phytates, lycopene, carotenoids, and other phytochemicals in vegetables may also have significant protective effects in reducing coronary risk [21].

Despite our attempt to control for several known confounding factors, it is still possible that the observed inverse association between fruits and vegetable intake and coronary risk could be at least partially explained by other factors associated with heart-healthy behaviours [22]. Also, in retrospective case-control studies two main sources of systematic errors may exist, the selection and the recall bias. In order to eliminate selection bias we set objective criteria both for patients and controls. However, insignificant misclassification may exist, since a small percentage of asymptomatic coronary patients may be wrongly assigned to controls, even they were evaluated by a cardiologist. Moreover, in case-control studies it is usually observed that patients who had a recent adverse event are more likely to place greater emphasis on several factors related to the disease than the control group (recall bias). To reduce this type of bias and analyse precise information, we obtained accurate information from the patients as well as from their relatives or their accompanying persons and compare their responses with Kendall's tau-criterion. Concerning the medical information we avoided recall 
bias by obtaining detailed data from subjects' medical records. However, over/under estimation of the relative risk may exist, especially due to the measurement of nutritional and smoking habits and the onset of the investigated cardiovascular risk factors. Moreover, the coronary patients who died at entry or the day after were not included into the study. This bias could influence our results. However, the proportion of deaths during the first two days was less than $4 \%$. Thus, it is unlikely that the exclusion of fatal events has significantly altered our findings. Finally, we tried to reduce the potential effect of uncontrolled or unknown confounders using the same study base, for patients and controls.

\section{Conclusion}

The presented scientific evidences are sufficient to give good reason for public health education and promotion aimed at a substantial increase in the consumption of fruits and vegetables. However, our data do not suggest evidences for causality and population trials are needed in order to provide scientific proof of their efficacy.

\section{Competing interests}

None declared.

\section{Authors' contributions}

DP: design of the study, statistical analysis and drafted the manuscript, CP: design of the study and drafted the manuscript, PK: editing, CC: drafted the manuscript, MV: editing, CS: conception of the study and editing, and PT: conception of the study and editing. All authors read and approved the final manuscript.

\section{Acknowledgment}

This study is supported by research grants from the Hellenic Heart Foundation (I I/1999-2002). The authors would like to thank the physicians and the specialists that coordinated the data collection of this study: Dr K Tzioumis (Athens, Crete, Pelloponisos), Dr J Skoumas (Athens), Dr N Papaioannou (Athens, Thessalia), Dr P Starvopodis (lonian Islands), Dr L Karra (Aegean Islands), Dr D Antoniades (Macedonia), Dr G Rembelos (Aegean Islands), Dr D Markou (Athens), A Moraiti (Athens), D Evagelou (Crete), Dr S Vellas (Attica, Hpeirous), Dr. G Skoumbourdis (Sterea Hellas), B Meidanis (Macedonia, Sterea Hellas, Thessalia), Dr S Loggos (Attica), Dr G Zavitsanakis (Sterea Hellas), Dr I Elefsiniotis (Athens), Dr A Theodorakis (Sterea Hellas), Dr N Marinakis (Aegean Islands), Dr T Kyratzoglou (East Macedonia) and S Zombolos (Peloponnese).

\section{References}

I. Wilson P, D'Agostino RB, Levy D, Belanger AM, Silbershatz $\mathrm{H}$ and Kannel WB Prediction of Coronary Heart Disease Using Risk Factor Categories Circulation 1998, 97: 1837-1847

2. Wood $D$ Established and emerging cardiovascular risk factors Am Heart J 200 I, I 41 :S49-57

3. Keys A, Mennoti A and Karvonen MJ The diet and 15-year death rate in the seven countries study Am J Epidemiol 1986, I24:903915

4. Trichopoulou A, Naska A and Vasilopoulou E Guidelines for the intake of vegetables and fruit: the Mediterranean approach Int J Vitam Nutr Res 200I, 7 I:149-53
5. Ness AR and Powles JW Fruit and vegetables, and cardiovascular disease: a review Int J Epidemiol 1997, 26: I-13

6. Law MR and Morris JK By how much does fruit and vegetable consumption reduce the risk of ischaemic heart disease? Eur J Clin Nutr 1998, 52:549-56

7. Boushey CJ, Beresford SAA, Omenn GS and Motulsky AG A quantitative assessment of plasma homocysteine as a risk factor for vascular disease: probable benefits of increasing folic acid intakes JAMA 1995, 274: 1049-57

8. Trichopoulou A From Research to Education: The Greek experience Nutrition 2000, 16:528-531

9. Trichopoulou A, Lagiou P and Trichopoulos D Traditional Greek diet and coronary heart disease J Cardiovasc Risk 1994, I:9-15

10. Panagiotakos DB, Pitsavos C, Chrysohoou C, Stefanadis C and Toutouzas PK Risk Stratification of Coronary Heart Disease, In Greece: Final Results from CARDIO2000 Epidemiological Study Prev Med 2002, 35:548-556

II. Panagiotakos DB, Pitsavos C, Chrysohoou C, Tzioumis K, Papaioannou I, Stefanadis C and Toutouzas PK The Association Of Mediterranean Diet With Lower Risk Of Acute Coronary Syndromes, In Hypertensive Subjects Int J Cardiol 2002, 82: I4I147

12. Pitsavos C, Panagiotakos DB, Chrysohoou C, Skoumas J, Papaioannou I, Stefanadis $C$ and Toutouzas PK The Benefits From Mediterranean Diet On The Risk Of Developing Acute Coronary Syndromes, In Hypercholesterolemic Subjects: A Case-Control Study (Cardio2000) Cor Artery Dis 2002, 1 3:295-300

13. Chrysohoou C, Panagiotakos DB, Pitsavos C, Kokkinos P, Marinakis $N$, Stefanadis $C$ and Toutouzas PK Gender Differences on the Risk Evaluation of Acute Coronary Syndromes: The CARDIO2000 Study Prev Cardiol 2003,

14. Willett W Nutritional Epidemiology New York: Oxford. University Press 1990,

15. Marchand JL, Luce D, Goldberg P, Bugel I, Salomon C and Goldberg $M$ Dietary factors and the risk of lung cancer in New Caledonia (South Pacific) Nutr Cancer 2002, 42(I): $18-24$

16. Van't Veer P, Jansen MC, Klerk M and Kok FJ Fruits and vegetables in the prevention of cancer and cardiovascular disease Public Health Nutr 2000, 3:103-107

17. Birkett NJ Intake of fruits and vegetables in smokers Public Health Nutr 1999, 2(2):217-22

18. Rimm EB, Stampfer M], Ascherio A, Giovannucci E, Colditz GA and Willett WC Dietary antioxidant intake and risk of coronary heart disease among men N Engl J Med 1993, 328: I450- I 456

19. American Heart Association Dietary Guidelines for healthy American Adults Circulation 1996, 94:1795-1800

20. Knekt $P$, Jarvinen $R$, Reunanen $A$ and Maatela J Flavonoid intake and coronary mortality in Finland: a cohort study BMJ 1996, 3 | 2:478-48|

21. Liua S, Leea IM, Ajania U, Colea SR, Buring JE and Mansona JA Intake of vegetables rich in carotenoids and risk of coronary heart disease in men: The Physicians' Health Study Int J Epidemiol 2001, 30:130-135

22. Nestle $M$ Fruits and vegetables: protective or just fellow travelers? Nutr Rev 1996, 54:255-257

Publish with Bio Med Central and every scientist can read your work free of charge

"BioMed Central will be the most significant development for disseminating the results of biomedical research in our lifetime. "

Sir Paul Nurse, Cancer Research UK

Your research papers will be:

- available free of charge to the entire biomedical community

- peer reviewed and published immediately upon acceptance

- cited in PubMed and archived on PubMed Central

- yours - you keep the copyright 